\title{
Downregulation of microRNA-34b is responsible for the elevation of blood pressure in spontaneously hypertensive rats
}

\author{
FAN YANG, HAIYU LI, YOUYOU DU, QIANGWEI SHI and LUOSHA ZHAO \\ Department of Cardiology, The First Affiliated Hospital of Zhengzhou University, Zhengzhou, Henan 450052, P.R. China
}

Received September 30, 2015; Accepted October 17, 2016

DOI: $10.3892 / \mathrm{mmr} .2017 .6122$

\begin{abstract}
The present study aimed to identify the microRNA (miRNA) responsible for the development of primary hypertension, and examine the downstream signaling pathway, which mediates the effect of the miRNA. Reverse transcription-quantitative polymerase chain reaction analysis was performed to identify which miRNA may be involved in the pathogenesis of hypertension. In silico analysis and a luciferase assay were used to validate the target of the selected miRNA, and miRNA mimics and small interfering (si)RNA of the target were transfected into smooth muscle cells to examine its effect on the biological activity of the cells. miR-34b was found to be upregulated in spontaneously hypertensive rats (SHRs), compared with Wistar Kyoto (WKY) rats. Therefore, the present study used online miRNA target prediction tools to predict the candidate target genes of miR-34b in the database, and consequently identified cyclin G1 (CCNG1) and cyclin-dependent kinase 6 (CDK6) as its possible target genes. CDK6 subsequently identified to be the direct target gene of miR-34b using a luciferase reporter assay in vascular smooth muscle cells (VSMCs). The present study also established the possible negative regulatory association between miR-34b and CDK6 via investigating the mRNA and protein expression levels of CDK6 and CCNG1 in VSMCs collected from the SHRs and WKY rats, respectively. To investigate the signaling pathways between miR-34b and CDK6, the mRNA and protein expression levels of CDK6, and the proliferation rates were compared in VSMCs transfected with CDK6 siRNA or miR-34b mimics, the results of which indicated that the miR-34b mimics exerted the same effects on the expression of CDK6 and cell proliferation as CDK6 siRNA. The negative regulatory association between miR-34b and its target, CDK6, was confirmed, which may offer potential as a novel therapeutic target in the treatment of hypertension.
\end{abstract}

Correspondence to: Dr Luosha Zhao, Department of Cardiology, The First Affiliated Hospital of Zhengzhou University, 1 Jianshe East Road, Zhengzhou, Henan 450052 P.R. China

E-mail: hypertensionmirna@126.com

Key words: microRNA-34b, spontaneously hypertensive rats, vascular smooth muscle cell, cyclin-dependent kinase 6 , proliferation

\section{Introduction}

The abnormal proliferation of vascular smooth muscle cells (VSMCs) is critical in several types of vascular disorders, including essential hypertension and atherosclerosis (1). The remodeled structure of the artery, with increased media and a smaller lumen, leads to cardiovascular complications, which are the predominant causes of hypertension-associated mortality (1). A number of regulators have been reported to be involved in the control of proliferation of VSMCs $(1,2)$. Investigations in this field are increasingly focused on the function of microRNAs (miRNAs) in VSMCs proliferation.

miRNAs are comprised of $\sim 22$ nucleotides, and are considered to be involved in the post-transcriptional regulation of mRNAs of its target genes by binding to sequences in the 3'-untranslated regions (3'UTRs) in the target mRNAs, leading to accelerated degradation of the target mRNA or repressed translation, and thereby causing a reduction in protein synthesis $(3,4)$. It has been reported that miRNAs are involved in several human pathophysiological and disease processes by controlling biological processes (4). Studies have demonstrated that miRNAs, including miRNA (miR)-143/145 (5-9), miR-221/222 (10,11) and miR-21 (12), are crucial in modulating the proliferation of VSMCs during the process of atherosclerosis and vascular injury. However, whether miRNAs are involved in modulating the proliferation of VSMCs in hypertension remains to be fully elucidated.

Accumulating evidence has shown that several specific miRNAs target the mRNAs of multiple genes, and function as regulators in the differentiation, apoptosis and proliferation of VSMCs (11,13-15). For example, miR-145 has been reported to target Krüppel-like factor 5, which is associated with the proliferation of VSMCs and has a regulatory effect on the phenotypic modulation of VSMCs (14). Other miRNAs, including miR-222 and miR-221, have been identified as novel regulators for neointimal hyperplasia and VSMC proliferation, by suppressing the expression of p57 (Kip2) and p27 (Kip1) (11). The findings may provide novel therapeutic targets to develop promising treatments for numerous proliferative vascular diseases, including hypertension and atherosclerosis.

Previously, high-throughput screening has been used to identify the candidate miRNAs with potential functional involvement in the pathogenesis of hypertension by comparing the miRNA expression profiles between Wistar Kyoto (WKY) rats and spontaneously hypertensive rats (SHRs) (16). 
Genetically, WKY rats and SHRs are from the same lineage, however, they have a notable difference in vascular phenotype (17). As the proliferation activity is markedly higher in the VSMCs of SHRs, compared with that of the VSMCs of WKY rats (17), the present study hypothesized that the different proliferation characteristics between SHR VSMCs and WKY rat VSMCs may be the major cause of the presence of hypertension in SHRs and the absence of hypertension in WKY rats. To confirm this hypothesis, the present study screened miRNAs, which have been reported to be aberrantly expressed in SHR VSMCs (16) and performed further investigations to identify the possible genes associated with these miRNAs.

\section{Materials and methods}

Cell culture. VSMCs were obtained from the medial layer of the thoracic aorta, which were collected from a total of 36 female SHRs and WKY rats (10-week-old), purchased from the Animal Centre of Zhengzhou University (Zhengzhou, China). The rats were maintained in a $12 \mathrm{~h}$ light/dark cycle at $25^{\circ} \mathrm{C}$ with food and water ad libitum. All animals were sacrificed using cervical dislocation The cultures were maintained at $37^{\circ} \mathrm{C}$ and a humidified atmosphere of $5 \% \mathrm{CO}_{2}$ in Dulbecco's modified Eagle's medium (DMEM) containing $10 \mathrm{U} / \mathrm{ml}$ streptomycin (Gibco; Thermo Fisher Scientific, Inc., Waltham, MA, USA), $10 \mathrm{U} / \mathrm{ml}$ penicillin (Gibco; Thermo Fisher Scientific, Inc.) and $10 \%$ fetal bovine serum (FBS; Hyclone; GE Healthcare Life Sciences, Logan, UT, USA). Cells in passages 3-6 were selected and used for experiments. The study protocol was approved by the Animal Ethical Committee at Zhengzhou University (Zhengzhou, China).

Cell culture and transfection. Human smooth muscle cells were obtained from the Chinese Academy of Sciences Cell Bank (Shanghai, China) and cultured in DMEM medium with $10 \% \mathrm{FBS}, 100 \mu \mathrm{g} / \mathrm{ml}$ streptomycin and $100 \mathrm{U} / \mathrm{ml}$ penicillin in a humidified atmosphere with $5 \% \mathrm{CO}_{2}$ at $37^{\circ} \mathrm{C}$, with cells in passages 3-8 used for further experiments. The inhibitor and mimic of miR-34b, and CDK6 small interfering (si)RNA were obtained from Guangzhou RiboBio Co., Ltd. (Guangzhou, China) with the following sequence: 5'-TACTTCTGAAGT GTTTGACATTT-3'. Cell transfection was performed using HiPerFect transfection reagent (Qiagen China Co., Ltd, Shanghai, China). The transfection complexes were added to the culture plates, cells were seeded at a density of $1.0 \times 10^{5}$, and incubated for $4 \mathrm{~h}$ at room temperature, following which the medium was replaced with fresh medium in accordance with the manufacturer's protocol.

$R N A$ preparation and reverse transcription-quantitative polymerase chain reaction ( $R T-q P C R)$ analysis. Total RNA was isolated from the cells using TRIzol reagent (Invitrogen; Thermo Fisher Scientific, Inc.). The Prime Script reverse transcription reagent kit (Takara Biotechnology Co., Ltd., Dalian, China) was used to perform the RT reaction with 0.2-0.5 $\mu \mathrm{g}$ RNA in order to detect miRNAs and mRNA, in accordance with the manufacturer's protocol. SYBR Premix Ex Taq (cat. no. DRR041A; Takara Biotechnology Co., Ltd.) was used to perform quantitative analysis to identify the expression levels of
miR-1, miR-34b, miR-500, miR-98, miR-72, and let-7a, and the mRNA expression levels of CCNG1 and CDK6, in an Applied Biosystems 7900 Real-Time PCR system (Applied Biosystems; Thermo Fisher Scientific, Inc.). The cDNA was amplified using qPCR using a SYBR green qPCR kit (Takara Bio, Inc., Otsu, Japan). The DNA polymerase was purchased from Takara Bio, Inc. The sequences of the primers were as follows: CDK6 sense, 5'-TGCACAGTGTCACGAACAGA-3'; antisense, 5'-ACCTCG GAGAAGCTGAAACA-3'; GAPDH sense, 5'-GATATTGTT GCCATCAATGAC-3'; U6 sense, 5'-CTCGCTTCGGCAGCA CA-3' and antisense, 5'-AACGCTTCACGAATTTGCGT-3'; and GAPDH sense, 5'-TGCACCACCAACTGCTTAGC-3' and antisense, 5'-GGCATGGACTGTGGTCATGAG-3'. The qPCR thermal cycling was performed as follows: Initial incubated for $15 \mathrm{sec}$ at $95^{\circ} \mathrm{C}$, followed by denaturing in 40 cycles at $95^{\circ} \mathrm{C}$ for $5 \mathrm{sec}$ and annealing for $31 \mathrm{sec}$ at $60^{\circ} \mathrm{C}$. The $2^{-\Delta \Delta \mathrm{Cq}}$ method (18) was applied to analyze the data. U6 was used to normalize the expression levels of mRNA and miRNAs as an internal control.

Western blot analysis. Cold phosphate-buffered saline (PBS) was used to wash the VSCMs three times, following which radioimmunoprecipitation assay buffer (Beyotime Institute of Biotechnology, Jiangsu, China) supplemented with phosphatase inhibitor cocktail and protease (Merck Millipore, Darmstadt, Germany) was used to lyse the cells. A Bradford assay (Bio-Rad Laboratories, Inc., Hercules, CA, USA) was performed to determine protein content. Subsequently, equal quantities of protein $(30 \mu \mathrm{g})$ were separated using $10 \%$ sodium dodecyl sulfate-polyacrylamide gel electrophoresis and then transferred onto a nitrocellulose membrane (EMD Millipore, Billerica, MA, USA). Membranes were blocked using PBS containing 3\% bovine serum albumin (Invitrogen; Thermo Fisher Scientific, Inc.) at room temperature for $60 \mathrm{~min}$. Primary antibodies were used to incubate the membranes at $4^{\circ} \mathrm{C}$ overnight following blocking. The antibodies comprised $\beta$-actin at a dilution of 1:1,000 (Santa Cruz Biotechnology, Inc., Dallas, TX, USA), and CCHG1 (cat. no. 3978) and CDK6 (cat. no. 13331) antibodies (Cell Signaling Technology, Inc., Danvers, MA, USA) at a dilution of 1:500. Horseradish peroxidase-conjugated anti-mouse or anti-rabbit secondary antibodies (cat. no. 7072; 1:1,000; Cell Signaling Technology, Inc.) were used to incubate the membranes for $1 \mathrm{~h}$ at room temperature. A chemiluminescence system (Cell Signaling Technology, Inc.) and ImageJ software (National Institutes of Health, Bethesda, MD, USA) were used to perform detection and relative density quantification.

Proliferation assay. A Cell Counting Kit-8 (CCK-8; Dojindo Molecular Technologies, Inc., Rockville, MD, USA) was used to count cell numbers according to the manufacturer's protocol. At $48 \mathrm{~h}$ post-transfection, the cells were collected and washed with PBS. Cells were seeded at a density of $2 \times 10^{6}$ and CCK-8 reagent (10 $\mu \mathrm{l})$ was added into each plate, followed by incubation at room temperature for $4 \mathrm{~h}$. A microplate reader was used to measure the absorbance at 450-540 nm (Multiskan Spectrum; Thermo Fisher Scientific, Inc.).

Luciferase reporter assay. The 3'UTRs of CDK6 and CCNG1 were amplified by qPCR using a SYBR green qPCR kit. The amplification was performed at $98^{\circ} \mathrm{C}$ for $60 \mathrm{sec}, 30$ cycles of 
$98^{\circ} \mathrm{C}$ for $30 \mathrm{sec}, 58^{\circ} \mathrm{C}$ for $30 \mathrm{sec}, 72^{\circ} \mathrm{C}$ for $2 \mathrm{~min}$ and $72^{\circ} \mathrm{C}$ for $5 \mathrm{~min}$. The PCR products were subsequently subcloned into a pGL3-basic vector (Invitrogen; Thermo Fisher Scientific, Inc.) carrying a firefly reporter gene. The reaction was performed in an $8 \mu \mathrm{l}$ mixture containing $100 \mathrm{ng}$ pGL3 basic vector, $200 \mathrm{ng}$ PCR products and placed in a $45^{\circ} \mathrm{C}$ water bath for $5 \mathrm{~min}$ and an ice bath immediately. Subsequently, $1 \mu 1$ 10xT4 ligase buffer and $1 \mu 12 \mathrm{mmol} / 1 \mathrm{ATP}$ were added, and incubated at $12^{\circ} \mathrm{C}$ overnight. The accuracy of the insert sequence was confirmed using direct Sanger sequencing. Furthermore, mutations were introduced into the constructs using site-directed mutagenesis (Stratagene, La Jolla, CA, USA).

The VSMCs were seeded into 24 -well plates (2x10 $/$ well) and, when the confluence reached $70-80 \%$, the cells were cotransfected with wild-type or mutant vectors and miR-34b mimics (Thermo Fisher Scientific, Inc.). After 48 h, the cells were assayed for luciferase activity using a Dual-Luciferase Reporter Assay system (Promega Corporation, Madison, WI, USA) according to the manufacturer's protocol. The Dual-Luciferase Reporter Assay system and GloMax 40/40 luminometer (Promega Corporation) were used to measure firefly luciferase activity according to the manufacturer's protocol.

Statistical analysis. MiRanda (www.microrna.org), TargetScan (www.targetscan.org) were used in the present study. All data are expressed as the mean \pm standard error of the mean. SPSS 16.0 (SPSS, Inc, Chicago, IL, USA) was used to perform one-way analysis of variance or Student's independent $t$-test to compare the parameters among groups. $\mathrm{P}<0.05$ was considered to indicate a statistically significant difference.

\section{Results}

miR-34b is differentially expressed in SHRs. To investigate the molecular mechanism of hypertension, the present study investigated miRNAs (miR-1, let-7a, miR-34b, miR-500, miR-98 and miR-72), and their downstream mediators and signaling pathways, as evidenced by observations that they are differentially expressed between SHRs and WKY rats. As shown in Fig. 1, the expression level of miR-34b in the SHRs was downregulated, compared with that in the WKY rats $(\mathrm{P}<0.05$; Fig. 1A), whereas the other mRNAs showed comparable levels of expression in the SHRs and WKY rats.

CDK6 is a target of $m i R-34 b$. The present study used online miRNA target prediction tools to predict the candidate target genes of miR-34b in the database, from which CCNG1 and CDK6 were identified as its possible target gene with the 'seed sequence' in the 3'UTR of CCNG1 (Fig. 2A) or CDK6 (Fig. 2B) separately. To identify the direct target gene of miR-34b, a luciferase reporter assay was performed in VSMCs, transfected with wild-type and mutant candidate target genes, respectively, and compared with scramble controls. As shown in Fig. 2C, the wild-type and mutant CCNG1 cells showed similar relative luciferase activity to that of the scramble control cells, indicating the mutant CCNG1 had no effect on the VSMCs. As shown in Fig. 2D, wild-type CDK6 shows showed lower relative luciferase activity, compared with the scramble controls $(\mathrm{P}<0.05)$, whereas the relative luciferase activity in the mutant CDK-6 group was

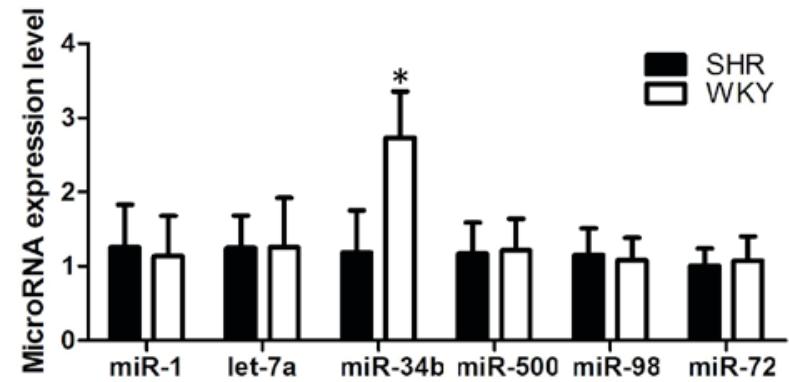

Figure 1. Expression levels of miR-1, let-7a, miR-34b, miR-500, miR-98 and miR-72 in SHR and WKY rat cell samples. Expression of miR-34b was upregulated in the SHRs, compared with the WKY rats, whereas the other microRNAs showed comparable expression levels in the SHRs and WKY rats, indicating miR-34b was involved in hypertensive disease. ${ }^{*} \mathrm{P}<0.05$ vs. WKY group. miR, microRNA; SHR, spontaneously hypertensive rats; WKY, Wistar Kyoto.

comparable to that of the scramble control, indicating CDK6 to be the target gene of miR-34b.

To further confirm CDK6 as the target gene of miR-34b, the present study investigated the mRNA and protein expression levels of CDK6 and CCNG1 in VSMCs collected from SHRs and WKY rats, respectively. As shown in Fig. 3, in the WKY rat VSMCs with overexpression of miR-34b, the mRNA expression levels of CCNG1 (Fig. 3B) and protein expression levels of CCNG1 (Fig. 3A) were similar between the two groups. By contrast, the mRNA (Fig. 3D) and protein (Fig. 3C) levels of CDK6 were downregulated in the WKY rats, compared with the SHRs $(\mathrm{P}<0.05)$, which confirmed CDK6 as the direct target gene of miR-34b, and indicate a possible negative regulatory association between miR-34b and CDK6.

Negative regulatory association between $\mathrm{miR}-34 \mathrm{~b}$ and its target, $C D K 6$. To investigate the signaling pathways between miR-34b and CDK6, the present study examined the mRNA and protein expression levels of CDK6 in VSMCs transfected with CDK6 siRNA or miR-34b mimics. As shown in Fig. 4, the mRNA levels of CDK6 (Fig. 4B) in the VSMCs transfected with the CDK6 siRNA or miR-34b mimics were comparably lower, compared with the scramble controls, indicating that the miR-34b mimics inhibited the expression of CDK6 in addition to CDK6 siRNA. The protein band of CDK6 (Fig. 4A; $\mathrm{P}<0.05$ ) in the miR-34b mimic group was lower in density, compared with that of the scramble control group, whereas the band of the CDK6 siRNA group showed comparable density with that of the miR-34b mimic group, indicating that the miR-34b mimics exhibited the same effect on the expression of CDK6 as CDK6 siRNA. The present study also examined the viabilities of the VSMCs in the groups. As shown in Fig. 5, the relative viability of the VSMCs transfected with miR-34b mimics was lower, compared with that of VSMCs in the scramble control group, and similar results were observed in the VSMCs transfected with CDK6 siRNA, confirming the negative regulatory association between miR-34b and its target, CDK6 $(\mathrm{P}<0.05)$.

\section{Discussion}

Accumulating evidence indicates that miRNAs are involved in vascular remodeling in cardiovascular diseases and in 
A

Hsa-miR-34b targets CCNG1

CCNG1 WT 3' UTR (143-150)

Hsa-miR-34b

5’-GTCTCAGACTTGGGAAAACTGCCTA-3'

3'-GUUAGUCGAUUACUGUGACGGAU-5'

CCNG1 Mut1 3' UTR (143-150)

5’-GTCTCAGACTTGGGAAATGACGGAT-3’

B

Hsa-miR-34b targets CDK6

CDK6 WT 3’ UTR (1089-1096)

Hsa-miR-34b

CDK6 WT 3' UTR (1089-1096)

C

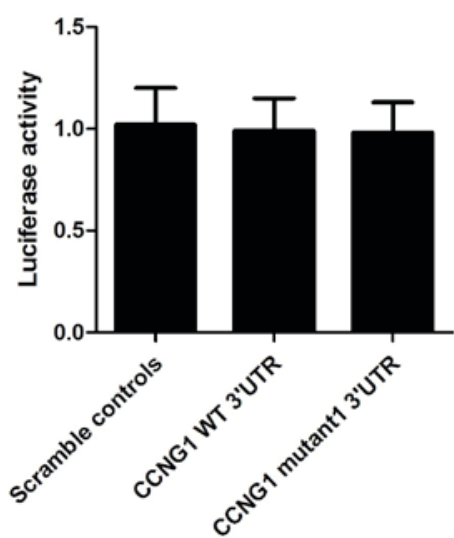

5’-CTGTACTTTCTGCCACACACTGCCTT-3’

3' -GUUAGUCGAUUACUGUGACGGAU-5'

5'-CTGTACTTTCTGCCACTGTGACGGAT-3’

D

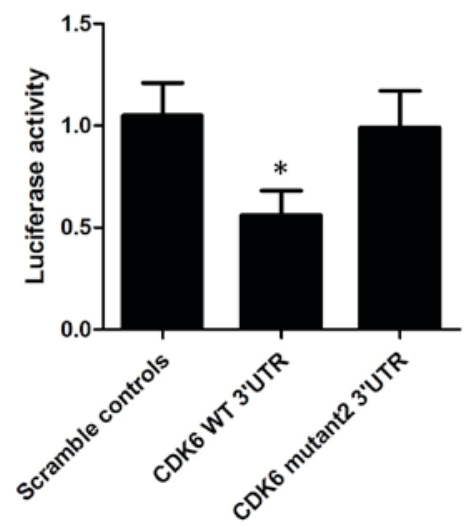

Figure 2. CDK6 as a target gene of miR-34b. (A) CCNG1 was identified as a possible target gene of miR-34b with the 'seed sequence' in the 3'UTR of CCNG1. (B) CDK6 was identified as a possible target gene of miR-34b with the 'seed sequence' in the 3'UTR of CDK6. (C) Results of the luciferase reporter assay in VSMCs showed the WT and mutant CCNG1 groups showed similar relative luciferase activity to the scramble controls, indicating the mutant in CCNG1 had no effect on VSMCs. (D) Results of the luciferase reporter assay in VSMCs showed the WT CDK6 group exhibited lower relative luciferase activity, compared with scramble controls. Luciferase activity in the mutant CDK6 group was comparable with that of the scramble controls, indicating CDK6 was a target gene of miR-34b. "P<0.05 vs. scramble control. CDK6, cyclin-dependent kinase 6; CCNG1, cyclin G1; miR, microRNA; 3'UTR, 3'untranslated region; VSMCs, vascular smooth muscle cells; WT, wild-type.

modulating VSMC proliferation. miR-146a, miR-26a, miR-24, miR-221/222 and miR-21 have been reported to directly induce the proliferation of VSMCs, which has been found to be mediated via platelet-derived growth factor and bone morphogenetic proteins in in vitro experiments $(3,12,13,19)$. However, the proliferation of VSMCs is inhibited by miR-145, miR-143 and miR-1 (12,20). The expression levels of miR-146a, miR-221/222 and miR-21 have been reported to be substantially elevated in balloon-injured carotid arteries in animal experiments, indicating the injury was attenuated by overexpression of these miRNAs $(3,19)$. Neointimal lesions in the femoral arteries of mice with hypertension or of old age can be induced by the deficiency of miR-143/145 (12). Other studies have reported that there is an upregulation of miR-125b in the VSMCs of diabetic mice (21), and there is abnormal vascularization of the retina when miR-218 is knocked down (22).
Clinical studies have shown that miR-145, which is abundant in smooth muscle, and miR-92a, miR-17 and miR-126, which are abundant in the endothelium, are significantly decreased in patients suffering from coronary artery disease, compared with healthy individuals (23). In the present study, it was found that the expression level of miR-34b in SHRs was upregulated, compared with that in WKY rats. CDK6, rather than CCNG1, was identified as the target gene of miR-34b using computational analysis and a luciferase assay.

miR-34b is a member of the miR-34 family, which is comprised of miR-34c, miR-34b and miR-34a. The physiological and pathophysiological importance of this family in human diseases, particularly in cancer, has been well documented; for example, the miR-34 family acts as a tumor suppressor by inducing cell-cycle arrest and apoptosis $(24,25)$. In particular, miR-34a represses the expression of pluripotency 
B

A

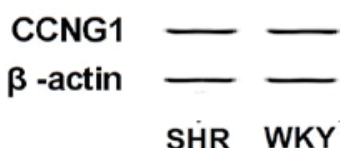

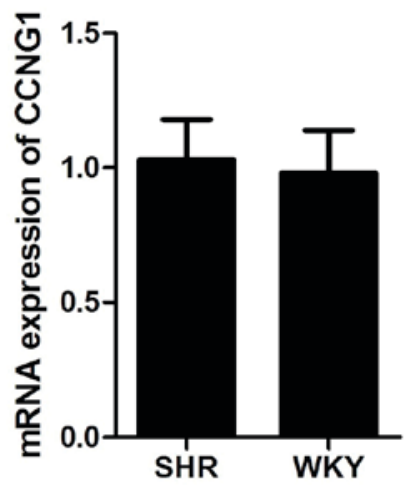

D

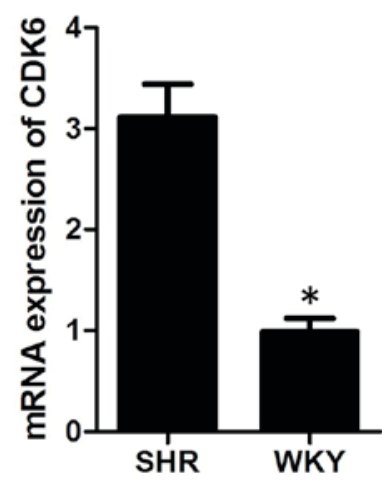

Figure 3. A negative regulatory association exists between miR-34b and CDK6. mRNA and protein expression levels of candidate target genes were examined. (A) Protein expression levels of CCNG1 were similar in the WKY rat and SHR VSMCs. (B) mRNA expression levels of CCNG1 were similar in the WKY rat and SHR VSMCs. (C) Protein expression levels of CDK6 were downregulated in the WKY rat VSMCs, compared with the SHR VSMCs. (D) mRNA expression levels of CDK6 were downregulated in the WKY rat VSMCs, compared with the SHR VSMCs. "P<0.05 vs. WKY group. CDK6, cyclin-dependent kinase 6; CCNG1, cyclin G1; miR, microRNA; VSMCs, vascular smooth muscle cells; WT, wild-type. SHR, spontaneously hypertensive rats; WKY, Wistar Kyoto.

A

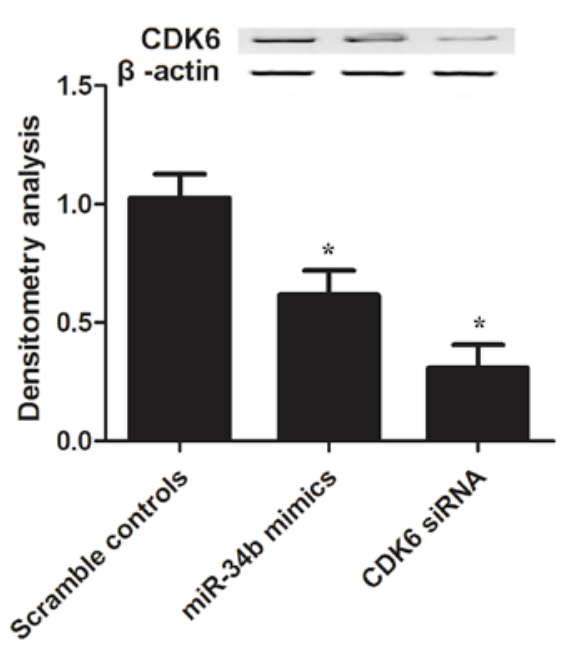

B

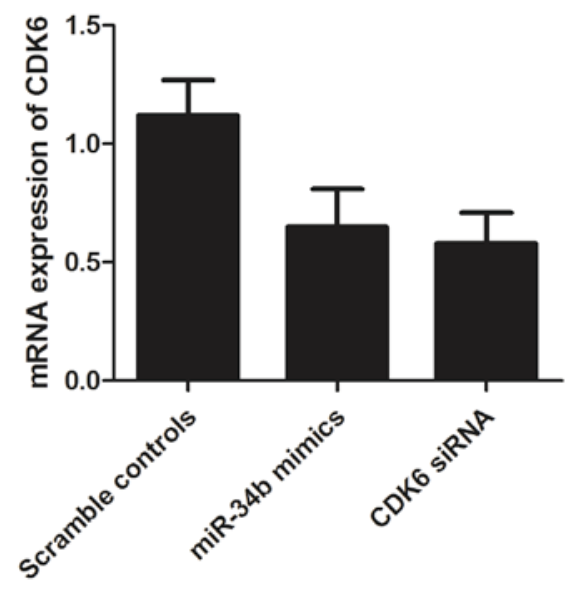

Figure 4. Confirmation of the negative regulatory association between miR-34b and CDK6 through investigation of signaling pathways. (A) Density of the CDK6 protein band in the miR-34b mimic group was lower, compared with that of the scramble control group. The density of the CDK6 siRNA band was comparable with that of the miR-34b mimic group, indicating the miR-34b mimics had the same effect on the expression of CDK6 as CDK6 siRNA. (B) mRNA levels of CDK6 in vascular smooth muscle cells transfected with CDK6 siRNA or miR-34b mimics were comparably lower, compared with that in the scramble controls, indicating the miR-34b mimics and CDK6 siRNA inhibited the expression of CDK6. ${ }^{*} \mathrm{P}<0.05$ vs. scramble control. CDK6, cyclin-dependent kinase 6; miR, microRNA; siRNA, small interfering RNA.

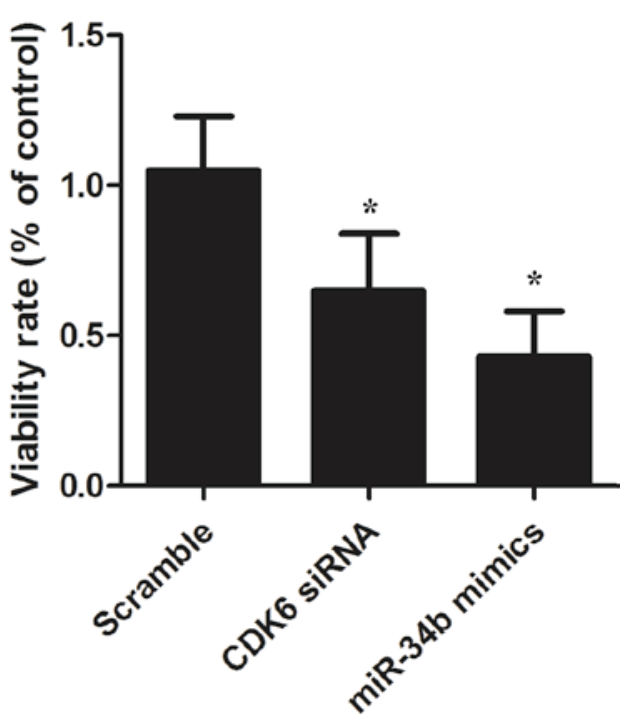

Figure 5. Assessment of cell viability. The relative viability of the VSMCs transfected with the miR-34b mimics was lower, compared with the scramble control VSMCs, and showed similar results to the VSMCs transfected with CDK6 siRNA. This confirmed the negative regulatory association between miR-34b and its target, CDK6. "P<0.05 vs. scramble control. CDK6, cyclin-dependent kinase 6; miR, microRNA; siRNA, small interfering RNA; VSMCs, vascular smooth muscle cells.

genes, including Mycn, (sex determining region Y)-box 2 and Nanog, which leads to the restriction of somatic reprogramming (26). The late steps of spermatogenesis are associated with miR-34c (27), and sperm-borne miR-34c is involved in modulating the expression of B cell lymphoma-2, which is critical for the control of cell division (28). The miR-34 family has been shown to be involved in the nervous system. miR-34c can act as a repressor of stress-induced anxiety by targeting stress-related corticotrophin releasing factor receptor type 1 and has a physiological function in regulating the central stress response (29). miR-34a targets silent information regulator 1 , which leads to regulation of the differentiation of neural stem cells from mice (30). In the present study, it was found that the mRNA and protein levels of CDK6 were downregulated in WKY rats, compared with SHRs, which confirmed CDK6 
as the direct target gene of miR-34b and indicated the possible negative regulatory association between miR-34b and CDK6. In addition, the present study found that the mRNA and protein expression levels of CDK6 in VSMCs transfected with CDK6 siRNA or miR-34b mimics were comparably lower, compared with levels in the scramble control cells, indicating that the miR-34b mimics, in addition to CDK6 siRNA, inhibited the expression of CDK6.

Cell proliferation is primarily controlled by the cell cycle, and progression of the cell cycle is predominantly regulated by cyclin-dependent kinases (CDKs) and cyclins. Cyclin D1 is a key protein in regulating the G1 phase and the cell cycle is more sensitive to alterations in cyclin D1, compared with other cyclins (31). CDK6 acts as a binding partner of cyclin D1, and its expression is critical for the entry of cells into the $S$ phase, which can be induced by the activated cyclin D1/CDK6 complex (32). In the present study, it was shown that the viability of VSMCs transfected with miR-34b mimics was lower, compared with the scramble controls, similar to the results of the VSMCs transfected with CDK6 siRNA. This confirmed the negative regulatory association between miR-34b and its target, CDK6.

Taken together, the present study demonstrated that miR-34b regulated the proliferation of VSMCs by inhibiting the expression of CDK6. The results of the present study, focused on miR-34b, provide further insight into the molecular mechanism of the development of hypertension, and improve current understanding of the pathogenesis of vascular remodeling in hypertension. However, further investigations are warranted to confirm the exact role of $\mathrm{miR}-34 \mathrm{~b}$ in the pathogenesis of hypertension in other models, including transgenic mice in which miR-34b is knocked down or overexpressed.

\section{References}

1. Owens GK, Kumar MS and Wamhoff BR: Molecular regulation of vascular smooth muscle cell differentiation in development and disease. Physiol Rev 84: 767-801, 2004.

2. Rzucidlo EM: Signaling pathways regulating vascular smooth muscle cell differentiation. Vascular 17 (Suppl 1): S15-S20, 2009.

3. Moazed D: Small RNAs in transcriptional gene silencing and genome defence. Nature 457: 413-420, 2009.

4. Krol J, Loedige I and Filipowicz W: The widespread regulation of microRNA biogenesis, function and decay. Nat Rev Genet 11: 597-610, 2010.

5. Boettger T, Beetz N, Kostin S, Schneider J, Krüger M, Hein L and Braun T: Acquisition of the contractile phenotype by murine arterial smooth muscle cells depends on the Mir143/145 gene cluster. J Clin Invest 119: 2634-2647, 2009.

6. Cordes KR, Sheehy NT, White MP, Berry EC, Morton SU, Muth AN, Lee TH, Miano JM, Ivey KN and Srivastava D: miR-145 and miR-143 regulate smooth muscle cell fate and plasticity. Nature 460: 705-710, 2009.

7. Xin M, Small EM, Sutherland LB, Qi X, McAnally J, Plato CF, Richardson JA, Bassel-Duby R and Olson EN: MicroRNAs miR-143 and miR-145 modulate cytoskeletal dynamics and responsiveness of smooth muscle cells to injury. Genes Dev 23: 2166-2178, 2009.

8. Deacon DC, Nevis KR, Cashman TJ, Zhou Y, Zhao L, Washko D, Guner-Ataman B, Burns CG and Burns CE: The miR-143-adducin3 pathway is essential for cardiac chamber morphogenesis. Development 137: 1887-1896, 2010.

9. Quintavalle M, Elia L, Condorelli G and Courtneidge SA: MicroRNA control of podosome formation in vascular smooth muscle cells in vivo and in vitro. J Cell Biol 189: 13-22, 2010.

10. Davis BN, Hilyard AC, Nguyen PH, Lagna G and Hata A: Induction of microRNA-221 by platelet-derived growth factor signaling is critical for modulation of vascular smooth muscle phenotype. J Biol Chem 284: 3728-3738, 2009.
11. Liu X, Cheng Y, Zhang S, Lin Y, Yang J and Zhang C: A necessary role of miR-221 and miR-222 in vascular smooth muscle cell proliferation and neointimal hyperplasia. Circ Res 104: 476-487, 2009.

12. Ji R, Cheng Y, Yue J, Yang J,Liu X, Chen H, Dean DB and Zhang C: MicroRNA expression signature and antisense-mediated depletion reveal an essential role of MicroRNA in vascular neointimal lesion formation. Circ Res 100: 1579-1588, 2007.

13. Chan MC, Hilyard AC, Wu C, Davis BN, Hill NS, Lal A, Lieberman J, Lagna $G$ and Hata A: Molecular basis for antagonism between PDGF and the TGFbeta family of signalling pathways by control of miR-24 expression. EMBO J 29: 559-573, 2010.

14. Elia L, Quintavalle M, Zhang J, Contu R, Cossu L, Latronico MV, Peterson KL, Indolfi C, Catalucci D, Chen J, et al: The knockout of miR-143 and -145 alters smooth muscle cell maintenance and vascular homeostasis in mice: Correlates with human disease. Cell Death Differ 16: 1590-1598, 2009.

15. Zhang C: MicroRNA-145 in vascular smooth muscle cell biology: A new therapeutic target for vascular disease. Cell Cycle 8: 3469-3473, 2009

16. Yu ML, Wang JF, Wang GK, You XH, Zhao XX, Jing Q and Qin YW: Vascular smooth muscle cell proliferation is influenced by let-7d microRNA and its interaction with KRAS. Circ J 75: 703-709, 2011.

17. Louis WJ and LG Howes: Genealogy of the spontaneously hypertensive rat and Wistar-Kyoto rat strains: Implications for studies of inherited hypertension. J Cardiovasc Pharmacol 16 (Suppl 7): S1-S5, 1990.

18. Livak KJ and Schmittgen TD: Analysis of relative gene expression data using real-time quantitative PCR and the 2(-Delta Delta C(T)) Method. Methods 25: 402-408, 2001.

19. Leeper NJ, Raiesdana A, Kojima Y, Chun HJ, Azuma J, Maegdefessel L, Kundu RK, Quertermous T, Tsao PS and Spin JM: MicroRNA-26a is a novel regulator of vascular smooth muscle cell function. J Cell Physiol 226: 1035-1043, 2011.

20. Xie C, Huang H, Sun X, Guo Y, Hamblin M, Ritchie RP, Garcia-Barrio MT, Zhang J and Chen YE: MicroRNA-1 regulates smooth muscle cell differentiation by repressing Kruppel-like factor 4. Stem Cells Dev 20: 205-210, 2011.

21. Villeneuve LM, Kato M, Reddy MA, Wang M, Lanting L and Natarajan R: Enhanced levels of microRNA-125b in vascular smooth muscle cells of diabetic db/db mice lead to increased inflammatory gene expression by targeting the histone methyltransferase Suv39h1. Diabetes 59: 2904-2915, 2010.

22. Small EM, Sutherland LB, Rajagopalan KN, Wang S and Olson EN: MicroRNA-218 regulates vascular patterning by modulation of Slit-Robo signaling. Circ Res 107: 1336-1344, 2010.

23. Fichtlscherer S, De Rosa S, Fox H, Schwietz T, Fischer A, Liebetrau C, Weber M, Hamm CW, Röxe T, Müller-Ardogan M, et al: Circulating microRNAs in patients with coronary artery disease. Circ Res 107: 677-684, 2010.

24. Hermeking H: The miR-34 family in cancer and apoptosis. Cell Death Differ 17: 193-199, 2010.

25. He L, He X, Lim LP, de Stanchina E, Xuan Z, Liang Y, Xue W, Zender L, Magnus J, Ridzon D, et al: A microRNA component of the p53 tumour suppressor network. Nature 447: 1130-1134, 2007.

26. Choi YJ, Lin CP, Ho JJ, He X, Okada N, Bu P, Zhong Y, Kim SY, Bennett MJ, Chen C, et al: miR-34 miRNAs provide a barrier for somatic cell reprogramming. Nat Cell Biol 13: 1353-1360, 2011.

27. Bouhallier F, Allioli N, Lavial F, Chalmel F, Perrard MH, Durand P, Samarut J, Pain B and Rouault JP: Role of miR-34c microRNA in the late steps of spermatogenesis. RNA 16: 720-731, 2010.

28. Liu WM, Pang RT, Chiu PC, Wong BP, Lao K, Lee KF and Yeung WS: Sperm-borne microRNA-34c is required for the first cleavage division in mouse. Proc Natl Acad Sci USA 109: 490-494, 2012.

29. Haramati S, Navon I, Issler O, Ezra-Nevo G, Gil S, Zwang R, Hornstein E and Chen A: MicroRNA as repressors of stress-induced anxiety: The case of amygdalar miR-34. J Neurosci 31: 14191-14203, 2011.

30. Aranha MM, Santos DM, Solá S, Steer CJ and Rodrigues CM: miR-34a regulates mouse neural stem cell differentiation. PLoS One 6: e21396, 2011.

31. Guan H, Zhu L, Fu M, Yang D, Tian S, Guo Y, Cui C, Wang L and Jiang H: 3,3'Diindolylmethane suppresses vascular smooth muscle cell phenotypic modulation and inhibits neointima formation after carotid injury. PLoS One 7: e34957, 2012.

32. Hotchkiss A, Robinson J, MacLean J, Feridooni T, Wafa K and Pasumarthi KB: Role of D-type cyclins in heart development and disease. Can J Physiol Pharmacol 90: 1197-1207, 2012. 